\title{
Effect of Reaction Temperature on Structural and Optical Properties of Reduced Graphene Oxide
}

\author{
Prerna Bansal, A. S. Panwar, and D. Bahadur
}

\begin{abstract}
Present study is aimed to see the influence of reaction temperature on the structural and optical properties of reduced graphene oxide powder samples. In the present work, aqueous solutions of graphite oxide (GO) were reduced at two different temperatures $\left(80\right.$ and $\left.95^{\circ} \mathrm{C}\right)$ using hydrazine hydrate. XRD and Raman studies showed that sample reduced at higher temperature has lesser defects. UV-visible spectral studies showed a blue shift in the absorption peak of GO after reduction due to the increased structural ordering because of restoration of $\mathbf{s p}^{2}$ carbon with temperature. More shift is observed for the sample reduced at higher temperature. Our study establishes a correlation between structural and optical properties of reduced graphene oxide powder samples with temperature.
\end{abstract}

Index Term - Graphite oxide (GO), reduced graphene oxide (RGO), photoluminescence (PL).

\section{INTRODUCTION}

Graphene, a single layer sheet of carbon atoms, has attracted great interest in recent years for its extraordinary mechanical, electrical, thermal and optical properties [1]-[3]. There are various approaches used for graphene synthesis. These approaches include micro-mechanical exfoliation of graphite, epitaxial growth, chemical vapor deposition (CVD) and chemical approaches like graphite oxidation and reduction and intercalation techniques [4]-[7]. Although first three methods can produce graphene with lesser defects and with better physical properties but chemical oxidation and reduction methods are advantageous in terms of high yield, solutions based processability, low cost and ease of implementation. We have synthesized graphite oxide (GO) from graphite powder using a recently proposed improved Hummer's method [8]. As synthesized graphite oxide (GO) is further reduced using hydrazine hydrate at two different reaction temperatures $\left(80\right.$ and $\left.95^{\circ} \mathrm{C}\right)$. Our experimental results suggest that higher reaction temperature leads to better reduction of GO giving reduced graphene oxide (RGO) with lesser defects.

\section{EXPERIMENTAL SECTION}

\section{A. Synthesis of Graphite Oxide (GO)}

An improved method [8] for the preparation of graphite

Manuscript received May 5, 2013; revised July 5, 2013. This work was supported by the Nanomission, Department of Science and Technology (DST), Government of India.

The authors are with the Department of Metallurgical Engineering and Material Science, IIT Bombay, India (e-mail: prerna.bansal@iitb.ac.in, dhirenb@iitb.ac.in, panwar@iitb.ac.in). oxide (GO) is used to oxidize pure Graphite (Sigma-Aldrich , $45 \mu \mathrm{m}, 99.99 \%$ ) for preparation of graphite oxide (GO) using Conc. $\mathrm{H}_{2} \mathrm{SO}_{4}$, Conc. $\mathrm{H}_{3} \mathrm{PO}_{4}$ and $\mathrm{KMnO}_{4}$ as oxidizing agent.

For the improved method, a 9:1 mixture of concentrated H2SO4/H3PO4 (60:6.66ml) was added to a mixture of graphite flakes (500mg, $1 \mathrm{wt}$ equiv.) and $\mathrm{KMnO} 4(3.0 \mathrm{~g}, 6 \mathrm{wt}$ equiv), producing a slight exotherm to $45-50^{\circ} \mathrm{C}$. The reaction was then heated to $55^{\circ} \mathrm{C}$ and stirred for 12 hours. The reaction was cooled to room temperature and then kept in an ice bath with $30 \% \mathrm{H} 2 \mathrm{O} 2(0.5 \mathrm{~mL})$. Further, it was filtered using Nylon filter paper with $0.2 \mu \mathrm{m}$ pore size in a vacuum filtration assembly. Remaining solid material was washed multiple times with water, $30 \% \mathrm{HCl}$, and ethanol through centrifugation $\left(7000 \mathrm{rpm}\right.$ for $15 \mathrm{~min}$. at $\left.12^{\circ} \mathrm{C}\right)$ and the supernatant was decanted away every time. The remaining material was coagulated with $35 \mathrm{ml}$ of diethyl ether and the resulting suspension was filtered again. The solid obtained on the filter paper was vacuum-dried overnight at $50^{\circ} \mathrm{C}$.

\section{B. Reduction of Graphene Oxide Using $\mathrm{N}_{2} \mathrm{H}_{4}$ at Two Different Reaction Temperatures $\left(80\right.$ and $95^{\circ} \mathrm{C}$ )}

As-synthesized graphite oxide (GO) (20mg) was dispersed in water $(100 \mathrm{ml})$ to create a $0.2 \mathrm{wt} \%$ dispersion [7]. Exfoliation of graphite oxide (GO) to graphene oxide was achieved by ultra-sonication of the dispersion in a bath sonicator for 1 hour. The obtained brown dispersion was kept for centrifugation (7000 r.p.m. at $12^{\circ} \mathrm{C}$ ) for 30 minutes to remove any unexfoliated graphite oxide. The resulting homogeneous GO dispersion was mixed with $8 \mu$ of hydrazine solution ( $80 \%$ pure) and kept for stirring in a flat bottom flask on a magnetic stirrer at $80^{\circ} \mathrm{C}$ for 6 Hours. Upon heating the brown dispersion turned into black precipitate. It was then filtered and resulting filtrate was dried overnight in a vacuum oven at $50^{\circ} \mathrm{C}$ giving RGO powder. To see the effect of temperature on the reduction of graphite oxide, same reduction protocol was repeated at $95^{\circ} \mathrm{C}$.

\section{RESUlt ANALYSIS AND DisCUSSION}

\section{A. XRD Results}

$\mathrm{X}$-ray diffraction (XRD) patterns for graphite oxide (GO) and reduced graphene oxide (RGO) powder samples are shown in Fig. 1. XRD study confirmed the reduction of graphite oxide with a shift of sharp diffraction peak of GO $\left(2 \theta \sim 9.56^{\circ}\right)$ to broad diffraction peak of reduced graphene oxide (RGO) at $2 \theta \sim 23^{\circ}$. It is observed that sample reduced at $95^{\circ} \mathrm{C}$ is more crystalline than the sample reduced at $80^{\circ} \mathrm{C}$, with the evolution of [100] peak.

From table I, it is observed that Peak position of [002] peak shifts towards higher ' $2 \theta$ ' values and ' $d$ ' spacing value was found to decrease with temperature. 


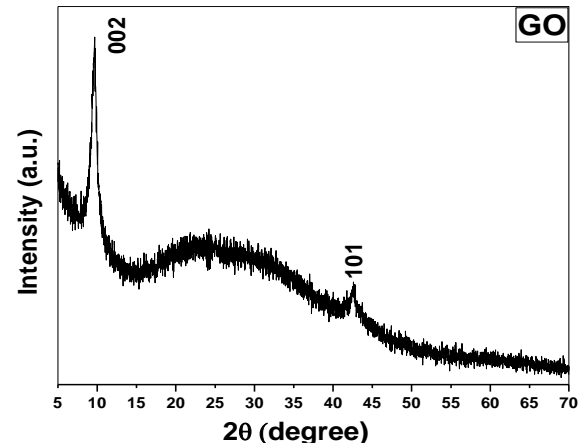

(a)

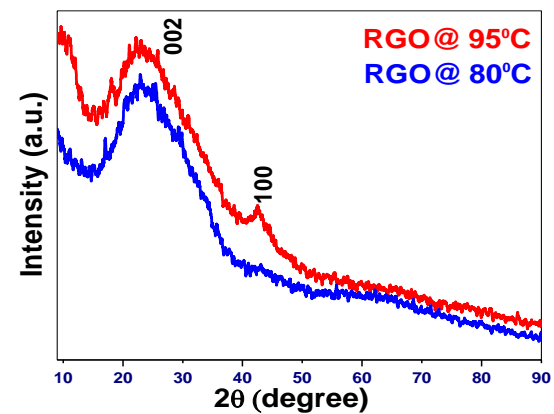

(b)

Fig. 1. XRD pattern of (a) GO and (b) RGO powder samples.

TABLE I EFFECT OF REACTION TEMPERATURE FOR [002] PEAK OF RGO

\begin{tabular}{|c|c|c|c|c|}
\hline $\begin{array}{c}\text { Reaction } \\
\text { Temperature } \\
\left({ }^{\circ} \mathrm{C}\right)\end{array}$ & $2 \theta\left(^{\circ}\right)$ & $\begin{array}{c}\text { FWHM } \\
\left({ }^{\circ}\right)\end{array}$ & $\begin{array}{c}\text { Plane } \\
\text { spacing } \\
\mathrm{d}(\AA)\end{array}$ & $\begin{array}{c}\text { Crystallite } \\
\text { size } \\
(\mathrm{nm})\end{array}$ \\
\hline 80 & 22.14 & 0.40 & 4.01 & 20.16 \\
\hline 95 & 22.28 & 0.53 & 3.98 & 15.12 \\
\hline
\end{tabular}

\section{B. Raman Spectra Analysis}

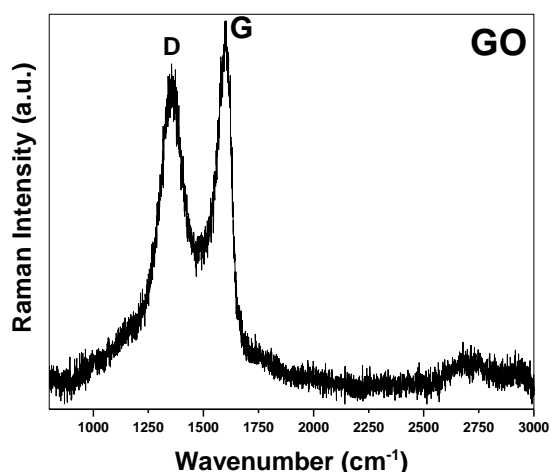

(a)

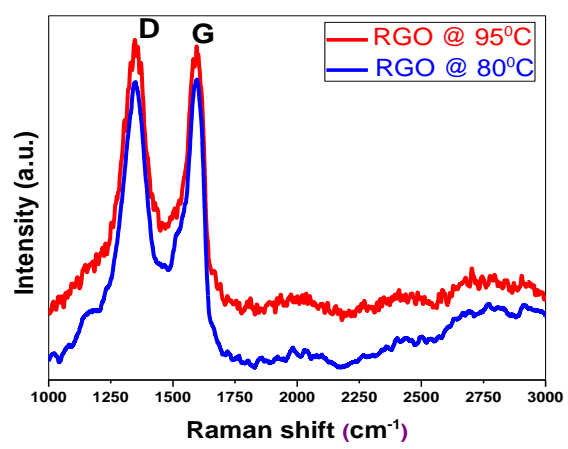

(b)

Fig. 2. Raman spectra of (a) GO and (b) RGO powder samples.
It is known that a Raman spectrum of pure graphite shows a strong $\mathrm{G}$ peak at $1570 \mathrm{~cm}^{-1}$ due to the first order scattering of $E_{2 \mathrm{~g}}$ modes. A broadened $\mathrm{D}$ peak is seen at $1350 \mathrm{~cm}^{-1}$ due to the reduction in size of in-plane $\mathrm{sp}^{2}$ domains in graphite induced by the creation of defects and distortions of the $\mathrm{sp}^{2}$ domains [7]. Fig. 2a shows the Raman spectra of GO with 'D' peak at $1349 \mathrm{~cm}^{-1}$ and ' $G$ ' peak at $1581 \mathrm{~cm}^{-1}$. Ratio of the ' $\mathrm{D}$ ' peak intensity to the ' $\mathrm{G}$ ' peak intensity $\left(\mathrm{I}_{\mathrm{D}} / \mathrm{I}_{\mathrm{G}}\right)$ for $\mathrm{GO}$ is $\sim 0.84$. Fig. $2 \mathrm{~b}$ shows Raman spectra of RGO prepared at $80^{\circ} \mathrm{C}$ and at $95^{\circ} \mathrm{C}$. $\mathrm{I}_{\mathrm{D}} / \mathrm{I}_{\mathrm{G}}$ ratio for $\mathrm{RGO}$ reduced at $95^{\circ} \mathrm{C}($ $\sim 1.32)$ is smaller than the $\mathrm{RGO}$ reduced at $80^{\circ} \mathrm{C}(\sim 1.41)$, indicating that the $\mathrm{sp}^{2}$ domain size increases at higher reaction temperatures.

\section{FTIR Results}

Fourier-transform infrared spectroscopy (FTIR) spectra is recorded for $\mathrm{GO}$ and $\mathrm{RGO}$ powder samples with $\mathrm{KBr}$ as reference. Fig. 3 presents the FTIR spectra of GO and RGO samples reduced at 80 and $95^{\circ} \mathrm{C}$.

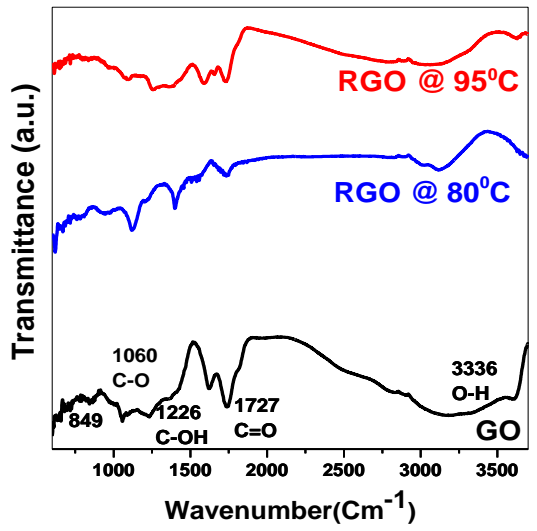

Fig. 3. FTIR spectra of GO and RGO powder samples.

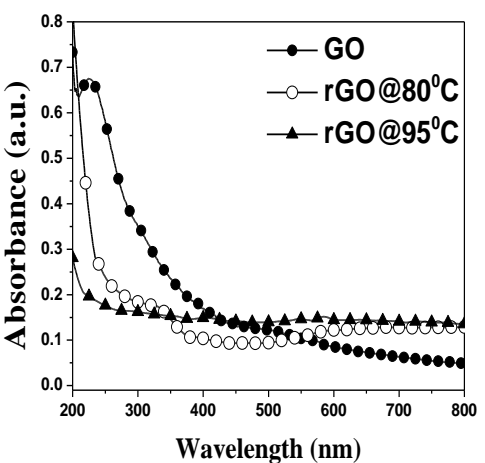

(a)

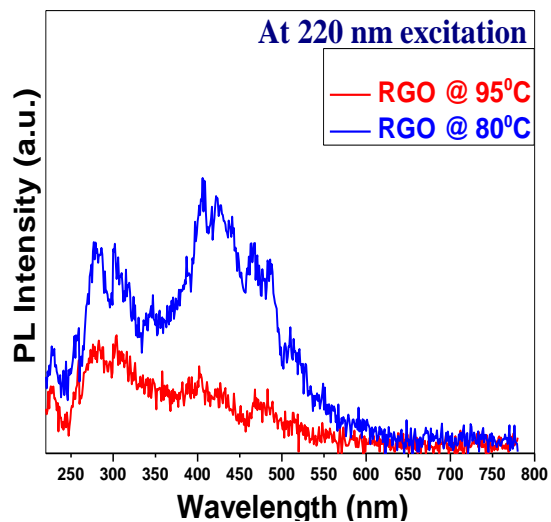

(b)

Fig. 4. (a) UV-vis absorption spectra and (b) PL spectra of GO and RGO. 
Fourier-transform infrared (FTIR) spectra of GO confirms the presence of sharp broad peak of intercalated free $\mathrm{H}_{2} \mathrm{O}$ molecules at nearly $3300 \mathrm{~cm}^{-1}$. Peaks corresponding to - $\mathrm{COOH}$ (carboxyl), -OH(hydroxyl), and $>\mathrm{O}$ (epoxide) groups are observed at $\sim 1740, \sim 1233$, and $\sim 1030 \mathrm{~cm}^{-1}$ respectively. FTIR spectra indicate the vaporization of intercalated free water molecules with temperature, as shown by the decrease of intensity of peaks correspond to - $\mathrm{OH}$ functional groups with temperature.

\section{Optical Studies}

Fig. 4 (a) shows the UV-visible spectra of the GO, showing a sharp absorption peak at $220 \mathrm{~nm}$, which is attributed to the $\pi-\pi *$ transitions of the $\mathrm{C}-\mathrm{C}$ aromatic rings [8]. After the reduction of GO, the absorption peak was blue shifted for both the RGO samples. Fig. 4(b) presents the room temperature photoluminescence (PL) spectra, which shows that the defect peak is suppressed in the sample reduced at higher temperature. Our UV study reveals that sample reduced at higher temperature has smaller optical gap. The significant blue shift is due to the increased structural ordering due to restoration of $\mathrm{sp}^{2}$ domains with temperature.

\section{CONCLUSIONS}

We have reduced aqueous solutions of GO at two different reaction temperatures $\left(80\right.$ and $\left.95^{\circ} \mathrm{C}\right)$ using hydrazine hydrate. XRD and Raman results were found in agreement with each other showing that higher reaction temperature leads to more crystalline nature of RGO sample with lesser defects. Optical studies suggest that due to the increased structural ordering with temperature, there is a blue-shift in the absorption spectra of RGO. Our study shows that crystalline nature, defects and optical properties of RGO depends on its reduction temperature.

\section{ACKNOWLEDGMENT}

The financial support from the Nanomission, Department of Science and Technology (DST), Government of India, is greatly acknowledged. We would like to acknowledge Sophisticated Analytical Instrument Facility (SAIF), IIT Bombay for characterization facilities.

\section{REFERENCES}

[1] K. Geim and K. S. Novoselov, "The rise of grapheme," Nature Materials, vol. 6, pp. 183-191, 2007.

[2] A. A. Balandin, "Thermal properties of graphene and nanostructured carbon materials," Nature Materials, vol. 10, pp. 569-581, 2011.

[3] K. S. Novoselov, A. K. Geim, S. V. Morozov, D. Jiang, Y. Zhang, S. V. Dubonos, I. V. Grigorieva, and A. A. Firsov, "Electric field effect in atomically thin carbon films," Science, vol. 306, pp. 666-669, 2004.

[4] X. S. Li, W. W. Cai, J. H. An, S. Kim, J. Nah, D. X. Yang, R. Piner, A Velamakanni, I. Jung, E. Tutuc, S. K. Banerjee, L. Colombo, and R. S. Ruoff, "Large-Area synthesis of high-quality and uniform graphene films on copper foils," Science, vol. 324, pp. 1312-1314, 2009.

[5] N. P. First, A. D. H. Walt, T. Seyller, C. Berger, J. A. Stroscio, and J. S. Moon, "Epitaxial graphenes on silicon carbide," MRS Bulletin, vol. 35, no.4, pp. 296-305, 2010.

[6] S. Malik, A. Vijayaraghavan, R. Erni, K. Ariga, I. Khalakhan, and J. P. Hill, "High purity graphenes prepared by a chemical intercalation method," Nanoscale, vol. 2, pp. 2139-2143, 2010.

[7] S Stankovich et al., "Synthesis of graphene-based nanosheets via chemical reduction of exfoliated graphite oxide," Carbon, vol. 45, pp. 1558-1565, 2007.

[8] D. C. Marcano, D. V. Kosynkin, J. M. Berlin, A. Sinitskii, Z. Z. Sun, A. Slesarev, L. B. Alemany, W. Lu, and J. M. Tour, "Improved synthesis of graphene oxide," ACS Nano, vol. 4, no. 8, pp. 4806-4814, 2010.

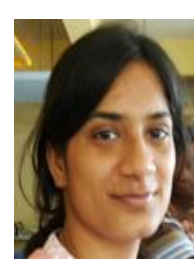

Prerna Bansal is a Ph.D. candidate (Materials Science) at department of Metallurgical Engineering and Material Science, IIT Bombay, India. She obtained her M.Tech. degree (Solid State Electronic Materials) from department of Physics, IIT Roorkee, India.

She is working on graphene-polymer composites and their possible applications. 